\title{
Imidazole-containing Cd Metal-Organic Framework with Selective Adsorption Properties
}

\author{
Yu-Ling Li, ${ }^{*}, 1$ Zin Zheng, ${ }^{2}$ Hui Nie, ${ }^{1}$ Chun-Mei Zhao, ${ }^{1}$ Yu-Fei Wang, ${ }^{1}$ Jing-Jing Li, ${ }^{1}$ Xiao-Li Zhou ${ }^{1}$ and Jia-Hui Zhang ${ }^{1}$ \\ ${ }^{1}$ School of Chemical Engineering and Food Science, Zhengzhou Institute of Technology, Zhengzhou 450044, China. \\ ${ }^{2}$ College of Science, Henan Agricultural University, Zhengzhou, Henan 450002, China.
}

\begin{abstract}
Metal-organic framework material $\left[\mathrm{Cd}(\mathrm{tib})(\mathrm{dnbpdc})\left(\mathrm{H}_{2} \mathrm{O}\right)\right] \cdot 2 \mathrm{DMF} \cdot 2 \mathrm{H}_{2} \mathrm{O}(\mathbf{1})[\mathrm{tib}=1,3,5$-tris $(1-$ imidazolyl)benzene, $\mathrm{H}_{2} \mathrm{dnbpdc}=2,2^{\prime}$-dinitro-4,4'-biphenyldicarboxylic acid] was synthesized and characterized by Thermogravimetric analyses (TGA), Powder X-ray diffraction (PXRD) analyses and Bruker D8 Advance X-ray diffractometer. The results showed that $\mathbf{1}$ was a $1 \mathrm{D}$ chain structure to be joined together by hydrogen bonds to generate a $3 \mathrm{D}$ supramolecular structure. $\mathrm{CO}_{2}$ and $\mathrm{N}_{2}$ adsorption behavior of the material was studied. It is significative that $\mathbf{1}$ can selective sorption of $\mathrm{CO}_{2}$.
\end{abstract}

\section{Introduction}

In the past decades, remarkable attention have been concentrated on the design and synthesis of functional metal-organic frameworks (MOFs) materials due to their rich and varied structures as well as potential application, such as gas storage and separation, magnetism, heterogeneous catalysis, photoluminescence and so on. [1-10]. Building MOFs with expected properties is an important task. However, it is still a challenge to investigate factors influencing the structural building of MOFs because varied factors can affect the structure and property of MOFs $[11,12]$. Such factors mainly include the selection of organic linkers, metal ions, reaction temperature. Among these factors, the organic linker is very crucial for formation of MOFs with various structures and properties. Therefore, proper organic linkers are significant in building the functional MOF materials. To our knowledge, 2,2'-dinitro-4,4'biphenyldicarboxylic acid $\left(\mathrm{H}_{2} \mathrm{dnbpdc}\right)$ may act as a good bridging ligand due to its various coordination modes and rigid multicarboxylic groups [13, 14].

In our previous studies, a rigid tridentate ligand 1,3,5-tris(1-imidazolyl)benzene (tib) with specific structure and interesting properties has been used in construction of MOFs [15-20]. Thus, it is meaningfull with imidazole-containing ligand tib and dicarboxylic acid $\mathrm{H}_{2}$ dnbpdc to construct MOFs with novel structures and properties. In this work, one new $\mathrm{Cd}$ metal-organic framework $\left[\mathrm{Cd}(\mathrm{tib})(\mathrm{dnbpdc})\left(\mathrm{H}_{2} \mathrm{O}\right)\right] \cdot 2 \mathrm{DMF} \cdot 2 \mathrm{H}_{2} \mathrm{O} \quad$ (1) [tib $=1,3,5$-tris (1-imidazolyl)benzene, $\mathrm{H}_{2} \mathrm{dnbpdc}=2,2^{\prime}$ dinitro-4,4'-biphenyldicarboxylic acid] was synthesized and characterized. Structural characterization revealed that 1 was a 1D chain structure to be joined together by hydrogen bonds to generate a 3D supramolecular structure. Gas adsorption property of the complex was studied. It is interesting that $\mathbf{1}$ shows selective and hysteretic sorption of $\mathrm{CO}_{2}$ over $\mathrm{N}_{2}$.

\section{Experimental}

\subsection{Materials and methods}

All commercially available chemicals and solvents are of reagent grade and were used as received without further purification. Ligand tib was prepared according to the procedures reported previously [21]. Elemental analyses for $\mathrm{C}, \mathrm{H}$ and $\mathrm{N}$ were performed on a FLASH EA 1112 elemental analyzer. Thermogravimetric analyses (TGA) were carried out on a Mettler-Toledo (TGA/DSC1) thermal analyzer under nitrogen with a heating rate of $10{ }^{\circ} \mathrm{C} \mathrm{min}^{-1}$. FT-IR spectra were recorded in the range of 400-4000 $\mathrm{cm}^{-1}$ on a BRUKER TENSOR 27 spectrophotometer with $\mathrm{KBr}$ pellets. Powder X-ray diffraction (PXRD) analyses were performed on a Bruker D8 Advance X-ray diffractometer with $\mathrm{Cu} \mathrm{K} \alpha(\lambda$ $=1.5418 \AA$ ) radiation. Sorption experiments were carried out on a Belsorp-max volumetric gas sorption instrument.

\subsection{X-ray crystallography}

Crystallographic data of $\mathbf{1}$ was collected on a Bruker Smart Apex II CCD area-detector diffractometer with graphite-monochromated Mo $\mathrm{K} \alpha$ radiation $(\lambda=0.71073$ $\AA$ ) using the $\omega$-scan technique. The diffraction data were integrated using the SAINT program [22], which was also used for the intensity corrections for the Lorentz and polarization effects. Semi-empirical absorption correction was applied using the SADABS program [23]. The structures were solved by direct methods with SHELXS-2014 and all the non-hydrogen atoms were

\footnotetext{
* Corresponding author: yulingli2013@163.com
} 
refined anisotropically on $F^{2}$ by the full-matrix leastsquares technique with the SHELXL-2014 [24, 25]. All the hydrogen atoms, except for those of water molecules, were generated geometrically and refined isotropically using the riding model. The hydrogen atoms of the coordinated water molecules were found from the Fourier map directly, while those of free water molecules were not found.

\subsection{Synthesis of complex 1}

A mixture of tib (13.8 mg, $0.05 \mathrm{mmol}), \mathrm{H}_{2} \mathrm{dnbpdc}(16.5$ $\mathrm{mg}, 0.05 \mathrm{mmol}), \mathrm{Cd}\left(\mathrm{NO}_{3}\right)_{2} \cdot 4 \mathrm{H}_{2} \mathrm{O}(30.8 \mathrm{mg}, 0.1 \mathrm{mmol})$ in $\mathrm{DMF} / \mathrm{H}_{2} \mathrm{O}(8 \mathrm{~mL}, \mathrm{v} / \mathrm{v}, 2: 1)$ was sealed in a $18 \mathrm{~mL}$ glass vial and heated at $90{ }^{\circ} \mathrm{C}$ for $72 \mathrm{~h}$, and then slowly cooled to room temperature. Light yellow block shaped crystals of 1 were obtained in $75 \%$ yield (based on $\left.\mathrm{H}_{2} \mathrm{dnbpdc}\right)$. Anal. Calcd for $1\left(\mathrm{C}_{35} \mathrm{H}_{38} \mathrm{~N}_{10} \mathrm{O}_{13} \mathrm{Cd}\right)$ : $\mathrm{C}$, 45.74; H, 4.17; N, 15.24\%. Found: C, 46.01; H, 4.39; N, 15.07\%. IR (KBr pellet, $\left.\mathrm{cm}^{-1}\right): 3396(\mathrm{~m}), 1664(\mathrm{~s}), 1614$ (s), $1527(\mathrm{~s}), 1400(\mathrm{~s}), 1345$ (s), 1253(m), $1073(\mathrm{~m})$, 1013 (m), 928 (m), 828 (m), 788 (m), 654 (w), $420(\mathrm{w})$.

\section{Results and discussion}

\subsection{Crystal structure description}

The results of X-ray crystallographic analysis show that 1 crystallizes in the triclinic $P-1$ space group. As shown in Fig. 3a, each $\mathrm{Cd}(\mathrm{II})$ is six-coordinated by three imidazole nitrogen, two carboxylate oxygen atoms and one coordinated water molecule. In 1 , each tib ligand links three $\mathrm{Cd}(\mathrm{II})$ atoms using its three imidazole groups to form an infinite 1D chain (Fig. 2). It is noteworthy that $\mathrm{dnbpdc}^{2-}$ acts as terminal ligand alternately coordinating at the up and down positions of the 1D chain (Fig. 2) in monodentate linkage mode. Furthermore, there are $\mathrm{C}-\mathrm{H} \cdots \mathrm{O}$ hydrogen bonding interactions between the neighbor $1 \mathrm{D}$ chains to generate 2D layer structure of $\mathbf{1}$ (Fig. 3) and 3D supramolecular framework of 1 (Fig. 4).

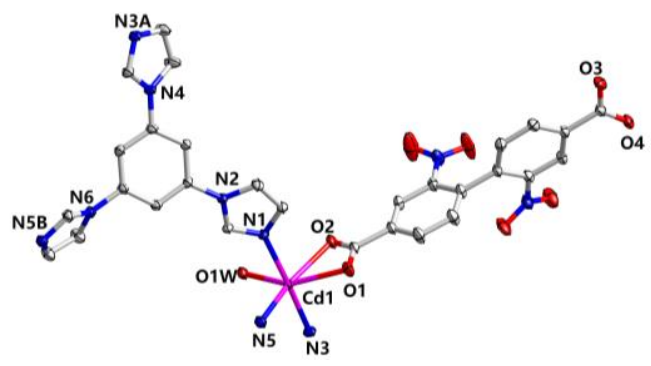

Fig. 1. Coordination environment of Cd(II) in 1. Hydrogen atoms and free solvent molecules are omitted for clarity.

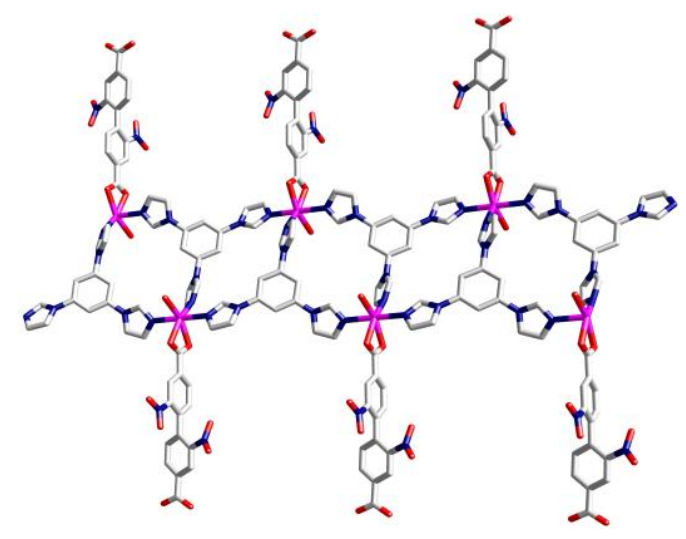

Fig. 2. 1D chain structure of $\mathbf{1}$.

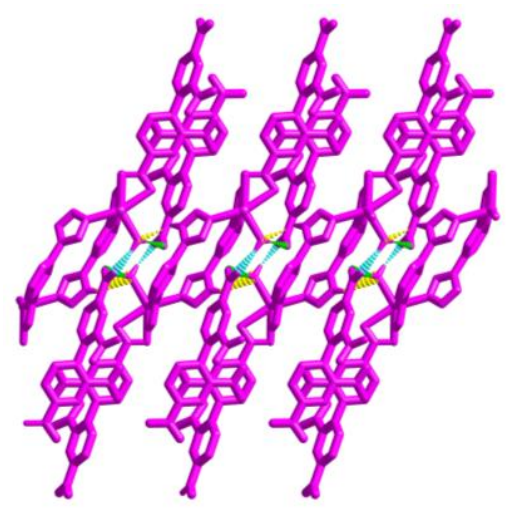

Fig. 3. 2D layer structure of $\mathbf{1}$ with hydrogen bonds indicated by dashed lines.

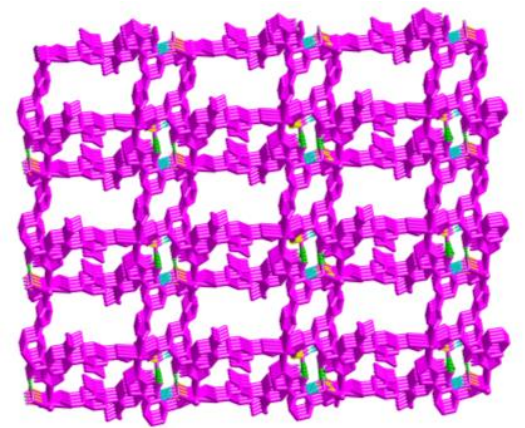

Fig. 4. 3D supramolecular structure of 1 with hydrogen bonds indicated by dashed lines.

\subsection{Powder X-ray diffraction (PXRD) and Thermogravimetric analysis (TGA)}

The pure phase of the synthesized 1 was confirmed by powder X-ray diffraction (PXRD) measurements and the results are shown in Fig. 5. Each PXRD pattern of the as-synthesized sample is consistent with the simulated one indicating the phase purity of the bulk samples.

Complex $\mathbf{1}$ is air stable and their thermal stability was investigated in the temperature range of $30-800{ }^{\circ} \mathrm{C}$ by TG measurements (Fig. 6). The TG curve of 1 displays a weight loss of $19.5 \%$ between $90-310{ }^{\circ} \mathrm{C}$, which corresponds to the loss of water and DMF molecules (calc. 19.8 wt.\%), and further weight loss was 
observed at about $360{ }^{\circ} \mathrm{C}$, owing to the collapse of the framework of $\mathbf{1}$.

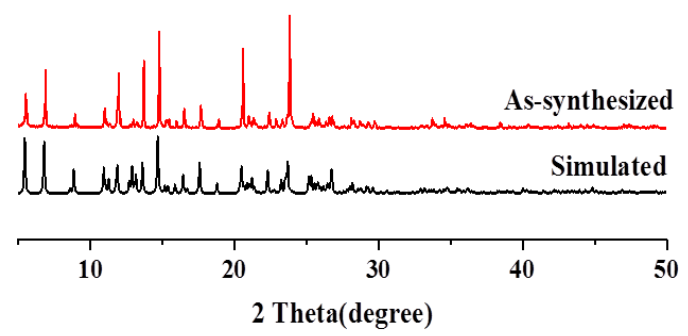

Fig. 5. PXRD pattern of 1.

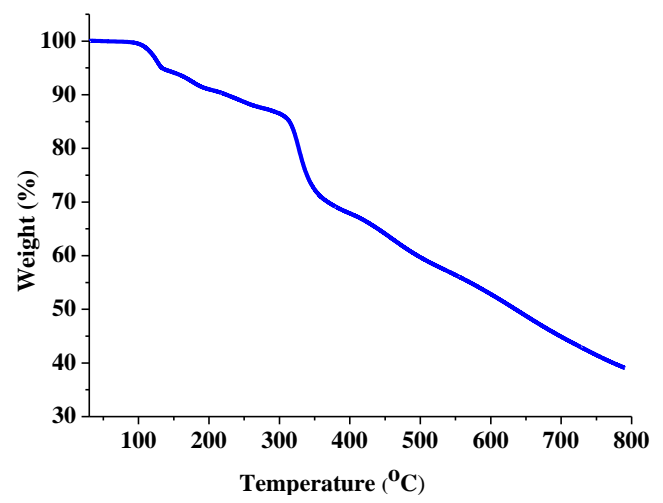

Fig. 6. TGA curve of $\mathbf{1}$.

\subsection{Gas and vapor adsorption properties.}

The porosity and high stability of the frameworks prompted us to examine their gas sorption behavior. The sorption performances of the activated samples $\mathbf{1}^{\prime}$ for $\mathrm{N}_{2}$ at $77 \mathrm{~K}, \mathrm{CO}_{2}$ at $195 \mathrm{~K}$ were discussed here. The activated sample $\mathbf{1}^{\prime}$ was prepared by immersing the assynthesized sample $\mathbf{1}$ in acetone for 3 days to remove the nonvolatile solvates, the solvent was decanted every $8 \mathrm{~h}$, and fresh acetone was added. The activated sample 1' was obtained by heating the solvent-exchanged sample at $423 \mathrm{~K}$ under a dynamic high vacuum for $10 \mathrm{~h}$.

As shown in Fig. 7 and Fig. 8, the sorption isotherms for 1' suggest that almost no adsorption of $\mathrm{N}_{2}$ at $77 \mathrm{~K}$. The final value of $\mathrm{CO}_{2}$ adsorption at $195 \mathrm{~K}$ is 32.2 $\mathrm{cm}^{3} \cdot \mathrm{g}^{-1}$ at $\mathrm{P}=0.99 \mathrm{~atm}$ corresponding to about $1.5 \mathrm{CO}_{2}$ molecules per formula unit for $\mathbf{1}^{\prime}$. The hysteresis and incomplete desorption suggests the strong interactions between the adsorbate and adsorbent [26], which includes the hydrogen bond interactions between the guest molecules and framework, as well as the guest molecules and the guest molecules. Given the above analysis results, we can see that complex 1 can select adsorption of $\mathrm{CO}_{2}$ molecules.

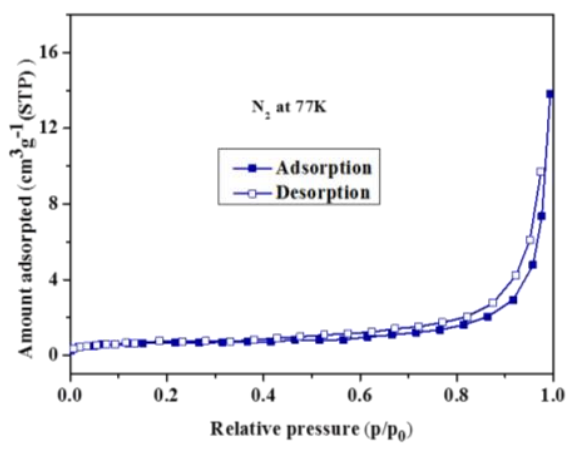

Fig. 7. $\mathrm{N}_{2}$ at $77 \mathrm{~K}$ sorption isotherms for $\mathbf{1}^{\prime}$. (Filled shape, adsorption; open shape, desorption).

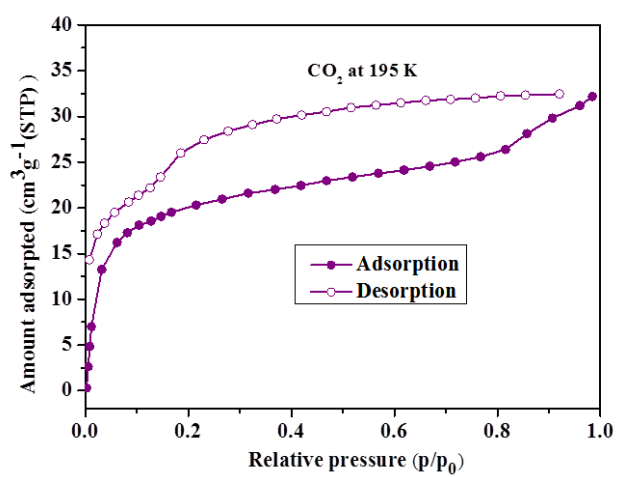

Fig. 8. $\mathrm{CO}_{2}$ at $195 \mathrm{~K}$ sorption isotherms for 1' (Filled shape, adsorption; open shape, desorption).

\section{Conclusions}

In conclusion, one new Cd (II)-based MOF with mixed ligands had been synthesized. By using dicarboxylate as the auxiliary ligand, we had obtianed one 1D chain structure. Furthermore, $\mathrm{N}_{2}$ and $\mathrm{CO}_{2}$ adsorption behavior of 1 was investigated and the results indicated that the frameworks showed the ability to selectively adsorb $\mathrm{CO}_{2}$, suggesting a possible application in selective gas adsorption and separation.

\section{Acknowledgements}

We gratefully acknowledge the Key Projects of Henan Provincial High School (grant no. 18A150042) for financial support of this work. This work was also financially supported by National Research Project Incubation Fund of Zhengzhou Institute of Technology (grant no. GJJKTPY2018K1).

\section{References}

1. X. L. Cui, K. J. Chen, B. L. Chen, et al. Science, $\mathbf{3 5 3}$ (2016) 141.

2. A. Karim, B. Youssef, E. Mohamed. Chem. Soc. Rev., 46 (2017) 3402.

3. Y. F. Wang, L. P. Wang, X. L. Zhou, Y. L. Li, J. J. Li. Journal of Molecular Structure, 1173 (2018), 612. 
4. B. Li, X. Dong, J. Li, et al. Nat. Commun., 8 (2017) 485.

5. D. Banerjee, C. M. Simonet, P. K. Thallapally, et al. Nat. Commun., 7 (2016) 11831.

6. J. Song, Z. Luo, D. K. Britt, H. Furukawa, O. M. Yaghi, K. I. Hardcastle, C. L. Hill, J. Am. Chem. Soc., 133 (2011) 16839.

7. W. M. Xuan, M. N. Zhang, Y. Liu, Z. J. Chen, Y. Cui, J. Am. Chem. Soc., 134 (2012) 690.

8. M. O'Keeffe, O. M. Yaghi, Chem. Rev., 112 (2012) 675.

9. Hughes, J. T.; Sava, D. F.; Nenoff, T. M.; Navrotsky. J. Am. Chem. Soc, 135 (2013) 16256.

10. G. Q. Kong, S. Ou, C. Zou, C. D. Wu, J. Am. Chem. Soc., 134 (2012) 1985.

11. L. S. Long, CrystEngComm, 12 (2010) 1354.

12. C. Hou, Q. Liu, J. Fan, Y. Zhao, P. Wang, W. Y. Sun, Inorg. Chem., 51 (2012) 8402.

13. Y. L. Li, D. Zhao, Y. Zhao, P. Wang, H. W. Wang, and W. Y. Sun, Dalton Trans., 45 (2016) 8816.

14. Y. L. Li, J-A Hua, Y. Zhao, Y. S. Kang, and W. Y. Sun, Micropor. Mesopor. Mater., 214 (2015) 188.

15. Z. Su, J. Fan, M. Chen, T. Okamura, W. Y. Sun, Cryst. Growth Des., 11 (2011) 1159.

16. Z. Su, J. Fan, M. Chen, T. Okamura, W. Y. Sun, N. Ueyamma, Cryst. Growth Des., 10 (2010) 3515.

17. C. Hou, Q. Liu, P. Wang, W. Y. Sun, Micropor. Mesopor. Mater. , 61 (2013) 61.

18. Y. L. Li, Y. Zhao, P. Wang, Y. S. Kang, Q. Liu, X. D. Zhang, and W. Y. Sun, Inorg. Chem., 55 (2016) 11821.

19. Y. L. Li, Y. Zhao, Y. S. Kang, X. H. Liu and W. Y. Sun, Cryst. Growth Des., 16 (2016) 7112.

20. Y. L. Li, Y. F. Wang, L. P. Zheng, X. L, Zhou, J. J. Li, (ICAEER 2018) E3S, Web of Conferences, 2018, 53, 01034.

21. J. Fan, L. Gan, H. Kawaguchi, W. Y. Sun, K. B. Yu, W. X. Tang, Chem.-Eur. J., 9 (2003) 3965.

22. SAINT, Program for Data Extraction and Reduction, Bruker AXS, Inc., Madison, WI, 2001.

23 G. M. Sheldrick, SADABS, University of Gottingen, Gottingen, Germany.

24 G. M. Sheldrick, SHELXS-2014, Program for the Crystal Structure Solution, University of Gottingen, Gottingen, Germany, 2014.

G. M. Sheldrick, SHELXL-2014, Program for the Crystal Structure Solution, University of Gottingen, Gottingen, Germany, 2014.
26. C. Hou, Q. Liu, Y. Lu, T. Okamura, P. Wang, M. Chen, W. Y. Sun, Micropor. Mesopor. Mater., 152 (2012) 96. 